\title{
Examination of the temporal effect in a flow injection analysis system using multi-channel absorbance detection
}

\author{
Shu-Hui Lin ${ }^{\mathrm{a}}$, Kai-Chieh Chang ${ }^{\mathrm{a}}$, Yu-Chun Chen ${ }^{\mathrm{a}}$, Tiing Yu ${ }^{\mathrm{a}, *}$, Su-Cheng Pai ${ }^{\mathrm{b}}$ \\ a Department of Applied Chemistry, National Chiao Tung University, Hsinchu 30050, Taiwan \\ b Division of Marine Chemistry, Institute of Oceanography, National Taiwan University, Taipei, Taiwan
}

\section{A R T I C L E I N F O}

\section{Article history:}

Received 10 July 2008

Received in revised form 17 October 2008

Accepted 17 October 2008

Available online 1 November 2008

\section{Keywords:}

Poiseuille effect

Temporal effect

Flow injection analysis

Tailing peaks

Asymmetry factor

Multi-channel detection

\begin{abstract}
A B S T R A C T
In addition to the Poiseuille effect, a so-called temporal effect was proposed recently to elucidate the commonly observed tailing peak signals of flow injection analysis (FIA). A multi-channel absorbance detector was used in this study to obtain the FIA peaks on both the spatial and temporal coordinates. The temporal effect was analyzed by comparison of the profiles between the experimental and the corresponding Gaussian peaks, and by comparison of asymmetry factors between the spatial and the temporal peaks. The temporal effect appeared to be the major factor under flow rates ranging from 0.5 to $8 \mathrm{ml} \mathrm{min}{ }^{-1}$. This was despite the presence of a spatially frontal peak observed in the FIA tubing, which was found to result in a tailing peak on the temporal coordinate due to this discussed cause. In addition, the temporal effect became greater as the flow rate increased.
\end{abstract}

(c) 2009 Published by Elsevier B.V.

\section{Introduction}

Since flow injection analysis (FIA) was first introduced in 1975 by Ruzicka and Hansen [1], it has been established as a simple, rapid, and versatile technique with widespread applications in quantitative analysis. This technique integrates the processes of sampling, reagent addition, mixing, and measurement in one system. It greatly enhances the operational efficiency for repetitive analysis. Unlike most chromatographic Gaussian-like signals, FIA peaks are usually asymmetric. Although the tailing of the peak may not be a big issue for analytical purposes, the rationale for this phenomenon draws researchers' attention. The FIA peaks have been studied by models including the exponentially modified Gaussian (EMG) function [2-5] and random walk models [6-8]. The tailed FIA peaks were usually elucidated by the Poiseuille effect which has been described frequently in papers and FIA books [9-13]. After injection of a sample plug, sample molecules begin moving forward in the tubing and supposedly develop into a symmetrical or Gaussian-like sample profile due to expansion. However, the dispersion of the sample plug in the tubing is the result of the redistribution of material through countless re-positioning of the elements of fluid in axial and radial directions caused by con-

\footnotetext{
* Corresponding author. Tel.: +886 3573 1673; fax: +88635723764

E-mail address: tyu@faculty.nctu.edu.tw (T. Yu).
}

vection and diffusion. The streamline, usually a laminar flow in small-bore tubing, is symmetric around the center of the channel where the fluid moves as rapidly as the mean flow velocity. Meanwhile, towards the walls, the velocity becomes much slower due to friction of the flow and the inner wall of the tubing. This parabolic flow profile due to the Poiseuille effect is believed eventually causing an FIA peak to become skewed, and thus the detector will record an asymmetrical peak. Ruzicka et al. [14-16] demonstrated graphically that the skewed profile obtained by the fixed-point detector reflected exactly how the concentration was distributed spatially in the FIA tubing. In addition to the Poiseuille effect, the junctions [17-19] of the FIA manifolds, such as at tubing connectors, sample injector, and detector flow cell, were considered to contribute to peak skewness.

iN ADDition to the Poiseuille effect, or named "spatial effect" in this work, another simple factor may have been ignored. Since the longitudinal dispersion of the sample distribution is very large in the FIA tubing, even a perfect Gaussian peak, which is observed spatially in the tube, would give a tailed peak monitored by a conventional single-channel detector. Therefore, the tailing peak occurs due to the non-simultaneous detection nature of using a fixed-position single-channel detector. The process for the peak tailing formation may be explained in Fig. 1. When a Gaussian peak just arrives at the detecting point, it has a relatively narrower width and greater peak height. As the peak keeps moving forward, it becomes broader and lower due to dispersion. 


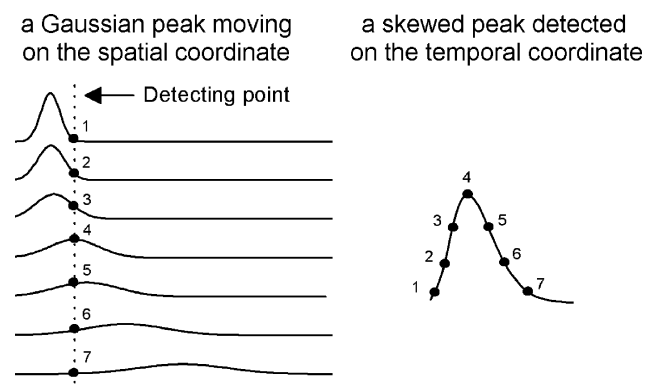

Fig. 1. Simulation of the temporal effect on peak tailing in FIA.

However, the detector monitors the signal sequentially as a function of time. As a result, the detected peak shows tailing even though the peak may look Gaussian on the spatial coordinate. This concept has been revealed by Glueckhauf [20] and further demonstrated using a worksheet practice [21]. It was also described by Giddings [22] and mentioned again recently by Felinger [23]. A mathematical treatment was carried out by Pai [24]. Pai investigated this observable fact using a temporally convoluted Gaussian (TCG) equation and named the cause for the tailing peak "temporal effect". The TCG equation simulated the peak profile under dispersion as the peak moving in the FIA tubing and thus resulted in an apparent tailing peak as usually observed for FIA. The conceptual approach of the TCG equation led to form a quite similar to the classical Taylor and Aris experiments [25-28], also close to that derived by Lapidus and Amundson [29]. The extent of the temporal effect in FIA could be determined by the difference between the TCG simulated curve and its corresponding Gaussian curve [24]. However, spatial profiles of FIA that can be used to directly contrast with the temporal profiles are still lacking.

Since the temporal effect occurs due to the non-simultaneous nature of the measurement using a single-channel detector; a multi-channel detection system was thus required to assess this effect. A two-channel on-column detection technique for monitoring solute dispersion in a capillary was developed by Evans and McGuffin in 1988 [30]. Rowlen et al. [31,32] then designed a multichannel detection system. Eleven discrete single-channel photon transducers were evenly installed on a chromatographic glass column to monitor the zone migrating along the chromatographic column during the separation process. This multi-channel detector was named a whole-column detection (WCD) system in their study. In fact, the WCD concept was first proposed in a theoretical study before it was fabricated [33]. Numerous whole-column imaging detection (WCID) systems have been developed in capillary electrophoresis (CE) for analyte imaging [34-36] using charge-coupled devises (CCD). In addition, direct photographic imaging was utilized by Shalliker et al. [37-39] to visualize band migration in liquid chromatography.

We designed a multi-channel absorbance detection system that allowed simultaneous absorbance measurements [40]. The peak profiles thus obtained were free from the temporal effect due to the system's capability of simultaneous monitoring for the whole FIA peak. Compared with the temporal peak profiles obtained using single-channel detection, we were able to determine the magnitude how the temporal effect would impinge on the peak skewness.

\section{Calculation for asymmetry factors}

In order to scrutinize the peak asymmetry for the spatial and temporal signals correlating with the temporal effect, the asymmetry factors [41] were calculated to compare the peak skewness in a more quantitative way. Given a tailing peak, as shown in Fig. 2, we

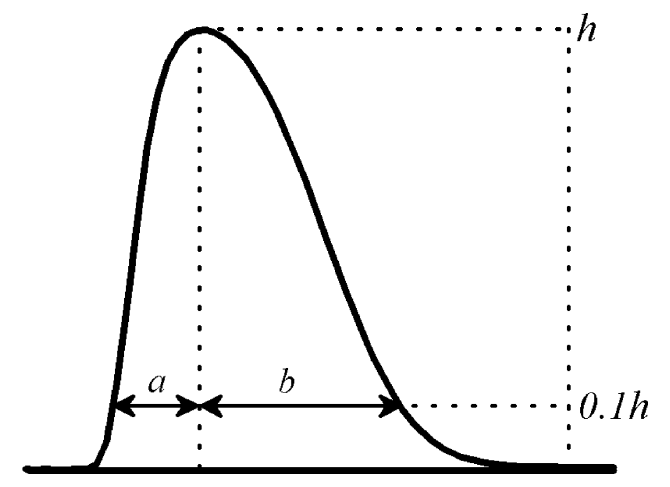

Fig. 2. Parameters for calculating asymmetry factor for a tailing peak.

located the peak summit position and found the point, $P_{1 / 10}$, at onetenth peak height. Then we extended from $P_{1 / 10}$ to both sides of the peak to hit upon two crossing points. We then measured components $a$ (the distance from the crossing point on the left-hand side of the curve to $P_{1 / 10}$ ) and $b$ (the distance from the crossing point on right-hand side of the curve to $\left.P_{1 / 10}\right)$. The asymmetry factor [41] for a temporal peak was defined as $A_{f}=(b-a) /(b+a)$. The range of $A_{f}$ was from -1 to 1 . For a tailing peak, $A_{f}$ was greater than 0 . As $A_{f}$ increased, the tailing phenomenon became larger. While $A_{f}$ was negative, a frontal peak would be observed. As for a perfect Gaussian peak, this factor would give a value of 0 . It was noted that while a "spatial" peak was passing through a fixed-position detecting point, its peak front (the right-hand side of the peak) appeared first, but when the whole peak passed the detecting point, the trace gave a temporal peak. The temporal peak front should, however, locate at the left-hand side of the peak. Accordingly, the temporal peak shape appeared like a mirror image of its corresponding spatial peak. The asymmetry factor for the spatial peak was thus defined as $A_{f}=(a-b) /(a+b)$ in order to make the comparison under the same basis. The asymmetry factor obtained for the temporal peak was denoted as $A_{f(t)}$, and that for the spatial peak was denoted as $A_{f(s)}$.

\section{Experimental}

\subsection{Multi-channel absorbance detection system}

A schematic diagram for the complete experimental set-up is displayed in Fig. 3. The multi-channel absorbance detection system was composed of a cold cathode fluorescence lamp $(21 \mathrm{~cm}$ long $\times 2 \mathrm{~mm}$ O.D.) as the light source, an optical cartridge to transfer the transmitted light to the embedded CCD with an observation range of $21 \mathrm{~cm}$. A $21 \mathrm{~cm} \times 2 \mathrm{~cm}$ interference filter (band center $=435 \mathrm{~nm}$, bandwidth $=50 \mathrm{~nm}$, transmittance $=90 \%$ ) was installed in front of the source to ensure a narrow bandwidth for absorbance measurements. The detailed description of the optical cartridge can be found elsewhere [40]. The alignment was adjusted using a translational stage to allow the source radiation to pass through the FIA tubing, the slit, the filter, and then enter the optical cartridge to make a linear image unto the CCD. The data were transmitted to a computer for further analysis. This multichannel absorbance detection system provided a $0.3 \mathrm{~mm}$ spatial resolution and a $3.6 \mathrm{~ms}$ temporal resolution. During each exposure period ( $3.6 \mathrm{~ms}$ ), the flow of the solute gave at most a $0.6 \mathrm{~mm}$ (calculated from the experiment with the fastest flow rate at $8 \mathrm{ml} \mathrm{min}^{-1}$ ) linear displacement in the tubing. This small movement could be considered negligible for peaks with very large widths recorded in FIA experiments. Thus, the moving peaks were considered "frozen" during the consecutive "snap shots." 


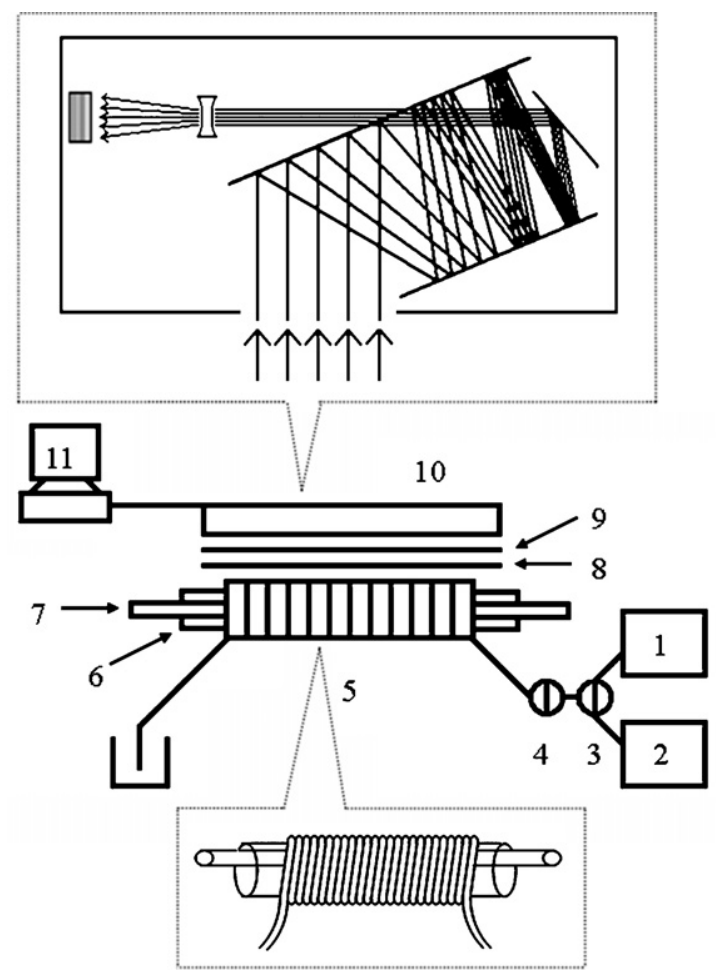

Fig. 3. Schematic diagram for the experimental set-up. 1: syringe pump; 2 : reciprocating pump; 3 : three-way valve; 4 : sample injection valve; 5 : coiled tubing wound from a glass tube (inset: detail for the coiled tubing); 6: glass tube; 7: light source; 8: slit; 9: interference filter; 10: optical cartridge for obtaining the $21 \mathrm{~cm}$ image (inset: detail for the optics in the cartridge); and 11: personal computer.

\subsection{Experimental procedures}

In the beginning of this study, a straight perfluoroalkoxy (PFA) tubing (1.0 mm I.D. $\times 1.6 \mathrm{~mm}$ O.D. purchased from Upchurch Scientific) was mounted at the optical path, and the absorbance signals were examined. However, the expansion of the injected sample during the FIA process was found to be much greater than $21 \mathrm{~cm}$, which was the measurement window of the multi-channel detector, during the FIA runs. Therefore, this detection system could not be applied to monitor an entire peak for peak shape analysis. A coiled PFA tubing, shown in the inset of Fig. 3, was then assembled to replace the straight tubing in order to allow full coverage of the sample peaks in the $21-\mathrm{cm}$ window. The PFA tubing was wound from a glass tube $(1.3 \mathrm{~cm}$ I.D. $\times 1.5 \mathrm{~cm}$ O.D.) in which the light tube was inserted. There were 108 coils at the $21-\mathrm{cm}$ detecting window. The light tube was installed near the side of the optical assembly in order to enhance the radiation intensity for the sample in the coiled tubing. The coil was connected to the sample injector on one end and to the waste on the other end. A syringe pump (ISCO Model 260D, Lincoln, NE, USA) and a reciprocating pump (Series II Digital HPLC Pump, Pharma-Tech Research Company, Baltimore, MD, USA) were connected to a Valco six-way sample injector via a three-way valve and then to the coiled tubing. The samples were injected through the six-way injector valve with a sample loop of $299.4 \mu \mathrm{L}$. The syringe pump was applied for analyzing sample peak profiles due to its accurate mass flow rate and pulse-free property. The reciprocating pump was used to deliver a pure sample solution during the calibration process. This arrangement allowed absorbance measurement for only a small portion of each coil. Five to six data points with different absorbances were obtained for each coil due to the variable pathlengths at the detectable portion of each coil when a sample solution was delivered into the coil. Since

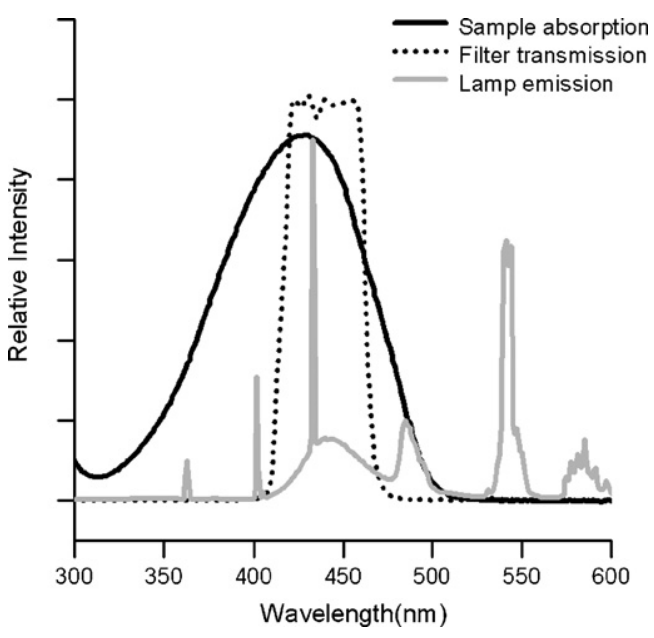

Fig. 4. The overlay diagram for the light source emission, the transmittance pattern of the filter, and the sample absorbance spectrum.

only the maximum absorbance for each coil was collected for further analysis, there were 108 data points in each measurement. In addition, the coiled tubing was $632 \mathrm{~cm}$ long, which was enough to cover several whole sample peaks even for a very large dispersion at higher flow rates. It was $\sim 39 \mathrm{~cm}$ from the sample injection valve to the first detecting point.

A solution of $1.0 \times 10^{3} \mathrm{mgl}^{-1}$ tartrazine (90\%, obtained from Sigma Chemical Co., St. Louis, MO, USA) was prepared using deionized water. The absorbance maximum was $429 \mathrm{~nm}$. The source emission spectrum, the transmittance pattern of the filter, and the sample absorbance spectrum are illustrated in Fig. 4. This combination ensured a high intensity and narrow bandwidth source for the absorbance measurements. The radiations from the light tube were first recorded as the background radiant powers $\left(P_{n}^{0}\right)_{n=1-108}$ at the 108 positions while the tube was eluted with de-ionized water under the desired flow rate. A sample was then injected. The radiant powers $\left(P_{n}\right)_{n=1-108}$ were measured again during the sample elution. The absorbance data was calculated using $\left(A_{n}\right)_{n=1-108}=$ $\log \left[\left(P_{n}^{0}\right)_{n=1-108} /\left(P_{n}\right)_{n=1-108}\right]$.

Magnitude calibration for the absorbance data was required. The 108 maximum absorbance data ( 1 for each coil) obtained were not equal when the dye solution was continuously delivered into the tubing. However, these absorbances must be considered equivalent (same concentration everywhere in the tubing) in order to analyze the peak when a sample plug was monitored during the FIA process. A calibration file was thus generated by dividing the individual photon counts of each data point by the average photon counts of the total data points in a run. This calibration file was used to treat all raw absorbance data for FIA measurements. Since the spatial peak pattern was recorded as a function of the longitudinal position of the tubing, the signals (the maximum absorbance for each coil) obtained must be calibrated for the precise location along the tubing. When the dye solution was delivered into the empty tubing at the desired flow rate, time $\left(t_{n}\right)$ was recorded at the appearance for the absorbance summit of each coil. The location $L_{n}$ for each summit could then be obtained by $L_{n=1-108}=Q t_{n=1-108} / a$, where $Q$ was the mass flow rate $\left(\mathrm{ml} \mathrm{min}^{-1}\right)$, and $a$ the tubing cross-section. In addition to the spatial peak profile, the temporal peak profile (peak signal recorded as a function of time) could also be obtained using the multi-channel detection system. Collecting the absorbance data as a function of time at a fixed longitudinal point gave the temporal peak at that point. 
Table 1

Experimental conditions and resultant data for spatial peaks.

\begin{tabular}{|c|c|c|c|c|c|c|}
\hline \multicolumn{3}{|c|}{ Experimental conditions } & \multicolumn{3}{|c|}{ Observed peak data } & \multirow{2}{*}{$\begin{array}{l}\text { Calculation } \\
A_{f(s)}\end{array}$} \\
\hline Peak no. & $Q\left(\mathrm{ml} \mathrm{min}^{-1}\right)$ & $L_{p}^{\prime}(\mathrm{cm})$ & $t^{\prime}(\mathrm{s})$ & $h^{\prime}(\mathrm{Abs})$ & $A_{L}^{\prime}(\mathrm{Abs}-\mathrm{cm})$ & \\
\hline 1 & 0.5 & 237.6 & $228.69(0.45)$ & $1.25(0.17)$ & $122.06(0.52)$ & $0.07(0.57)$ \\
\hline 2 & 0.5 & 274.7 & $263.95(0.57)$ & $1.22(0.20)$ & $123.21(0.51)$ & $0.06(0.64)$ \\
\hline 3 & 0.5 & 312.3 & $297.03(0.50)$ & $1.20(0.12)$ & $125.18(0.60)$ & $0.06(0.61)$ \\
\hline 4 & 0.5 & 354.3 & $336.71(0.44)$ & $1.19(0.10)$ & $127.92(0.89)$ & $0.05(0.71)$ \\
\hline 5 & 0.5 & 397.6 & $377.53(0.23)$ & $1.17(0.05)$ & $130.30(1.25)$ & $0.05(0.77)$ \\
\hline 1 & 1 & 237.6 & $115.75(0.35)$ & $1.09(0.49)$ & $127.10(0.55)$ & $0.06(0.70)$ \\
\hline 2 & 1 & 274.7 & $133.35(0.29)$ & $1.05(0.62)$ & $127.88(0.72)$ & $0.04(0.81)$ \\
\hline 3 & 1 & 312.3 & $150.26(0.53)$ & $1.01(0.33)$ & $129.19(0.79)$ & $0.04(0.83)$ \\
\hline 4 & 1 & 354.3 & $169.12(0.49)$ & $0.98(0.33)$ & $130.92(0.70)$ & $0.04(0.76)$ \\
\hline 5 & 1 & 397.6 & $189.95(0.20)$ & $0.96(0.35)$ & $132.68(0.72)$ & $0.03(0.64)$ \\
\hline 1 & 2 & 237.6 & $59.00(0.32)$ & $0.92(0.88)$ & $131.72(1.23)$ & $-0.03(1.22)$ \\
\hline 2 & 2 & 274.7 & $67.90(0.40)$ & $0.87(1.11)$ & $132.65(1.30)$ & $-0.03(1.40)$ \\
\hline 3 & 2 & 312.3 & $75.83(0.23)$ & $0.83(1.10)$ & $133.84(1.37)$ & $-0.03(1.35)$ \\
\hline 4 & 2 & 354.3 & $84.73(0.41)$ & $0.80(1.13)$ & $135.29(1.35)$ & $-0.02(1.44)$ \\
\hline 5 & 2 & 397.6 & $95.52(0.31)$ & $0.77(1.06)$ & $136.64(1.36)$ & $-0.02(1.36)$ \\
\hline 1 & 4 & 237.6 & $31.99(0.13)$ & $0.74(1.42)$ & $129.04(1.29)$ & $-0.11(1.42)$ \\
\hline 2 & 4 & 274.7 & $36.32(0.18)$ & $0.68(1.21)$ & $130.43(1.27)$ & $-0.10(1.33)$ \\
\hline 3 & 4 & 312.3 & $39.71(0.21)$ & $0.65(1.28)$ & $131.47(1.26)$ & $-0.10(1.38)$ \\
\hline 4 & 4 & 354.3 & $43.52(0.19)$ & $0.62(1.34)$ & $132.44(1.23)$ & $-0.09(1.38)$ \\
\hline 5 & 4 & 397.6 & $49.25(0.23)$ & $0.60(1.39)$ & $133.84(1.21)$ & $-0.08(1.41)$ \\
\hline 1 & 8 & 237.6 & $16.76(0.28)$ & $0.72(1.48)$ & $128.14(1.46)$ & $-0.15(1.61)$ \\
\hline 2 & 8 & 274.7 & $19.23(0.30)$ & $0.65(1.37)$ & $130.21(1.45)$ & $-0.16(1.56)$ \\
\hline 3 & 8 & 312.3 & $21.09(0.24)$ & $0.60(1.40)$ & $131.74(1.53)$ & $-0.14(1.59)$ \\
\hline 4 & 8 & 354.3 & $22.94(0.22)$ & 0.57 (1.39) & $133.30(1.53)$ & $-0.14(1.57)$ \\
\hline 5 & 8 & 397.6 & $26.32(0.25)$ & $0.53(1.44)$ & $135.58(1.51)$ & $-0.14(1.60)$ \\
\hline
\end{tabular}

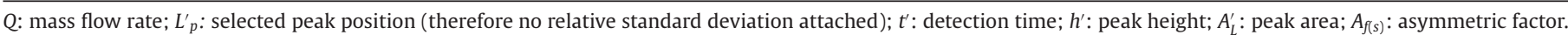
The relative standard deviations of three repetitive experiments are listed in parentheses.

Table 2

Experimental conditions and resultant data for temporal peaks.

\begin{tabular}{|c|c|c|c|c|c|c|c|}
\hline \multicolumn{3}{|c|}{ Experimental conditions } & \multicolumn{3}{|c|}{ Observed peak data } & \multicolumn{2}{|l|}{ Calculation } \\
\hline Peak no. & $Q\left(\mathrm{ml} \mathrm{min}^{-1}\right)$ & $D_{p}(\mathrm{~cm})$ & $t_{p}^{*}(\mathrm{~s})$ & $h^{*}(\mathrm{Abs})$ & $A_{t}{ }^{*}$ (Abs-s) & $A_{f(t)}$ & $D^{*}\left(\mathrm{~cm}^{2} \mathrm{~s}^{-1}\right)$ \\
\hline 1 & 0.5 & 237.6 & $227.95(0.44)$ & $1.29(0.28)$ & $115.39(0.48)$ & $0.14(0.60)$ & $2.37(1.18)$ \\
\hline 2 & 0.5 & 274.7 & $262.86(0.52)$ & $1.26(0.23)$ & $117.00(0.58)$ & $0.13(0.66)$ & $2.26(1.13)$ \\
\hline 3 & 0.5 & 312.3 & $297.40(0.13)$ & $1.24(0.23)$ & $118.06(0.90)$ & $0.13(0.63)$ & $2.12(1.52)$ \\
\hline 4 & 0.5 & 354.3 & $338.18(0.55)$ & $1.24(0.25)$ & $121.92(0.41)$ & $0.11(0.60)$ & $2.04(1.34)$ \\
\hline 5 & 0.5 & 397.6 & $378.26(0.52)$ & $1.22(0.11)$ & $124.60(0.70)$ & $0.11(0.67)$ & $1.96(2.75)$ \\
\hline 1 & 1 & 237.6 & $114.44(0.51)$ & $1.12(0.21)$ & $60.60(0.34)$ & $0.18(0.53)$ & $7.38(1.12)$ \\
\hline 2 & 1 & 274.7 & $131.15(0.17)$ & $1.07(0.66)$ & $60.55(0.99)$ & $0.17(0.61)$ & $7.19(1.10)$ \\
\hline 3 & 1 & 312.3 & $149.82(0.29)$ & $1.03(0.22)$ & $60.09(0.50)$ & $0.16(0.50)$ & $6.72(0.93)$ \\
\hline 4 & 1 & 354.3 & $170.21(0.28)$ & $1.02(0.24)$ & $61.97(0.09)$ & $0.13(0.51)$ & $6.53(0.16)$ \\
\hline 5 & 1 & 397.6 & $190.60(0.43)$ & $0.99(0.34)$ & $63.01(0.83)$ & $0.12(0.60)$ & $6.40(0.62)$ \\
\hline 1 & 2 & 237.6 & $57.60(0.30)$ & $0.95(0.75)$ & $31.26(0.82)$ & $0.21(0.94)$ & $22.80(0.32)$ \\
\hline 2 & 2 & 274.7 & $66.74(0.76)$ & $0.89(0.68)$ & $31.61(0.68)$ & $0.19(1.06)$ & $23.25(0.42)$ \\
\hline 3 & 2 & 312.3 & $75.14(0.35)$ & $0.85(0.61)$ & 31.39 (1.38) & $0.19(0.97)$ & $22.65(1.33)$ \\
\hline 4 & 2 & 354.3 & $85.14(0.51)$ & $0.83(0.59)$ & $32.32(0.62)$ & $0.16(0.86)$ & $22.59(1.04)$ \\
\hline 5 & 2 & 397.6 & $95.29(0.17)$ & $0.80(0.55)$ & $32.72(1.16)$ & $0.14(0.94)$ & $22.41(1.42)$ \\
\hline 1 & 4 & 237.6 & $30.19(0.74)$ & $0.76(0.92)$ & $14.92(1.08)$ & $0.27(1.57)$ & $64.53(0.99)$ \\
\hline 2 & 4 & 274.7 & $34.36(0.35)$ & $0.69(1.20)$ & $15.36(1.14)$ & $0.24(1.75)$ & $71.42(1.57)$ \\
\hline 3 & 4 & 312.3 & $38.48(0.94)$ & $0.67(1.18)$ & $15.24(1.53)$ & $0.23(1.82)$ & 68.27 (2.97) \\
\hline 4 & 4 & 354.3 & $43.41(0.56)$ & $0.64(1.37)$ & $15.68(1.75)$ & $0.22(1.84)$ & $70.29(0.31)$ \\
\hline 5 & 4 & 397.6 & $48.95(0.25)$ & $0.61(1.43)$ & $15.98(1.72)$ & $0.17(1.70)$ & $72.50(0.64)$ \\
\hline 1 & 8 & 237.6 & $15.21(0.32)$ & $0.75(1.31)$ & $7.65(1.24)$ & $0.37(1.64)$ & $135.85(4.43)$ \\
\hline 2 & 8 & 274.7 & $16.96(0.93)$ & $0.69(1.46)$ & $7.84(1.91)$ & $0.34(1.79)$ & $154.38(1.42)$ \\
\hline 3 & 8 & 312.3 & $19.64(0.65)$ & $0.61(1.64)$ & $7.73(1.17)$ & $0.32(1.73)$ & $168.84(0.74)$ \\
\hline 4 & 8 & 354.3 & $22.73(0.86)$ & $0.58(1.37)$ & $8.04(1.00)$ & $0.27(1.61)$ & 175.53 (3.17) \\
\hline 5 & 8 & 397.6 & $25.60(0.52)$ & $0.55(1.51)$ & $8.16(1.21)$ & $0.23(1.62)$ & $179.14(1.37)$ \\
\hline
\end{tabular}

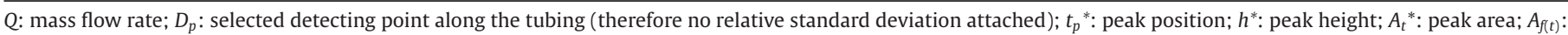
asymmetric factor. The relative standard deviations of three repetitive experiments are listed in parentheses. $D^{*}$ : dispersion coefficient. 


\section{Results and discussion}

\subsection{The temporal effect affected by dispersion under different flow rates}

The experiments were conducted under five different flow rates, $0.5,1,2,4$, and $8 \mathrm{ml} \mathrm{min}^{-1}$, respectively. For each flow rate, three repetitive experiments were made to confirm the reproducibility. All the experimental conditions, observed data, and calculated asymmetry factors for the spatial peaks are listed in Table 1, while those for temporal peaks are listed in Table 2. Although higher flow rates appeared to give less reproducible data, the relative standard deviations were within $2.0 \%$ for all relevant parameters. For each flow rate, five spatial peaks with their mass centers reaching at the tubing positions $237.6,274.7,312.3,354.3$, and $397.6 \mathrm{~cm}$ were chosen for further analysis. The typical spatial peaks collected, with flow rate $=1 \mathrm{ml} \mathrm{min}{ }^{-1}$, are shown in Fig. 5(a). Their corresponding temporal peak profiles could then be obtained by collecting the absorbance data at those five positions as a function of time. The temporal peaks thus obtained are shown in Fig. 5(b). As the spatial peak propagated from the tube inlet to the outlet, it became wider and lower due to the longer time allowed for dispersion. The trend for temporal peak broadening looked similar, i.e. the bandwidth became greater as time elapsed. While the spatial peaks
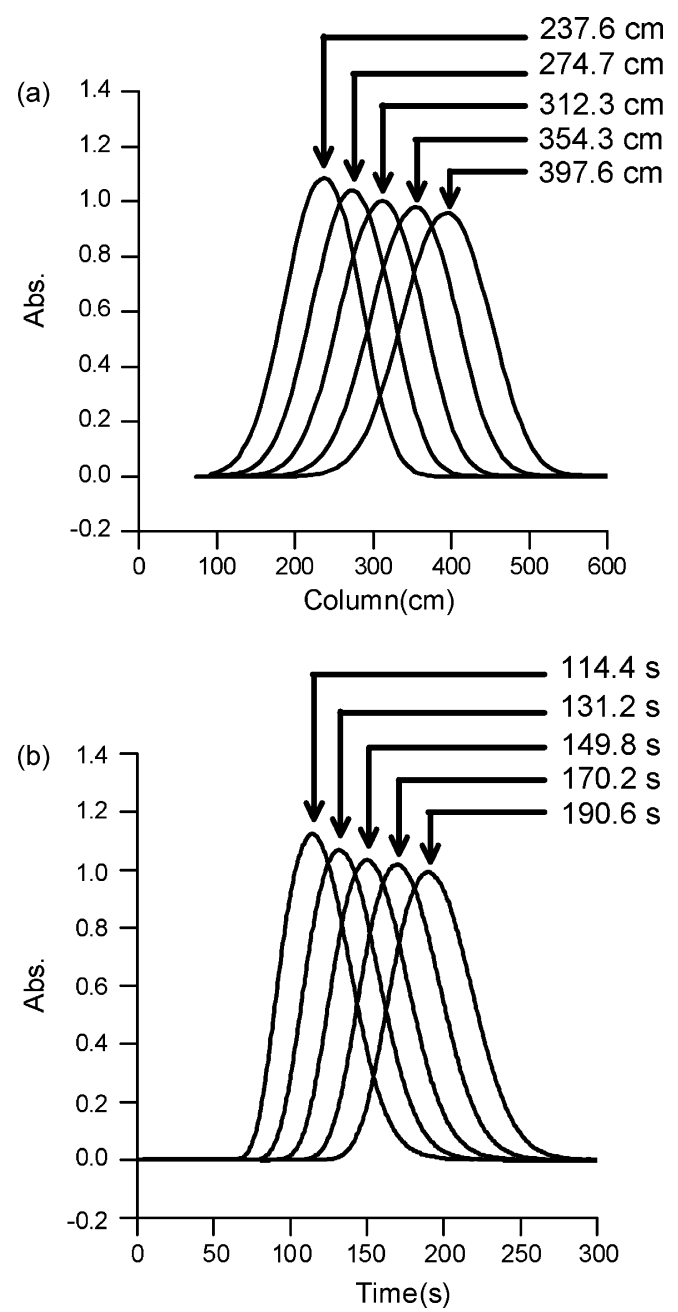

Fig. 5. Typical spatial peaks (flow rate $=1 \mathrm{ml} \mathrm{min}^{-1}$ ) acquired at five longitudinal positions (a); and their corresponding temporal peaks acquired at the same longitudinal positions with the peak summits labeled in time (b) during the FIA process. looked essentially symmetric, their temporal counterparts showed apparent tailing.

In addition, a higher flow rate caused a wider broadening on the spatial coordinate (see Table 1 ). According to the concept illustrated in Fig. 1, if the peak width on the spatial coordinate dispersed greater during the elution, the temporal effect upon the FIA signal on the time coordinate appeared more pronounced. The peak width is apparently related to dispersion. In fact [25,42], dispersion occurred due to the effective diffusion that arises from randomly sampling streamlines with different velocities. The deviation of the peaks from Gaussian profile could occur under higher flow rates. The dispersion coefficient $D^{*}\left(\mathrm{~cm}^{2} \mathrm{~s}^{-1}\right)$ can be estimated by the temporal data, using the equation recently derived by Pai et al. [43]:

$D *=\frac{A_{t}^{* 2} u^{2}}{4 \pi h^{* 2} t_{p}^{*}}-\frac{\sigma_{L 0}^{2}}{2 t_{P}^{*}}$

where $A_{t}^{*}$ is the peak area, $u$ the average flow speed, $h^{*}$ the peak height, $t_{p}^{*}$ the peak position, and $\sigma_{L O}$ the standard deviation of the sample initial state. The calculated results are listed in Table 2. The dispersion coefficients thus obtained are in the same order of magnitude compared with the results in the literature $[43,44]$. The temporal effect (judged by asymmetry factors) increased with dispersion under higher flow rate, as observed in Table 2.

\subsection{The temporal effect analyzed using the temporal data alone}

Pai [24] defined the magnitude of the temporal effect as the difference between the observed temporal peak profile and the corresponding Gaussian profile. Each experimental temporal peak provides three basic peak parameters $\left(t_{p}^{*}, A_{t}^{*}\right.$ and $\left.\sigma_{t}^{*}\right)$. With the parameters, an experimental peak can be restored to a Gaussian peak as a function of time, $t$, using the following equation [24]:

$C(t)=\frac{A_{t}^{*}}{\sqrt{2 \pi} \sigma_{t}^{*}\left(t_{p}^{*}\right)} \mathrm{e}^{-\left(t-t_{p}^{*} / \sqrt{2} \sigma_{t}^{*}\left(t_{p}^{*}\right)\right)^{2}}$

In order to quantitatively determine the extent of temporal effect, the original recorder track obtained at $312.3 \mathrm{~cm}$ as a function of time was overlapped with its restored Gaussian peak, shown in Fig. 6 . The residue curve (the Gaussian peak subtracting from the experimental peak) for each peak pair was plotted below and had been normalized as \% of the peak height. The negative quantity on the left-hand side and the positive quantity on the right-hand side for the residue curve illustrated that the experimental peak resulted in a tailing shape from Gaussian. Under higher flow rates, the difference between the two curves became larger. The amplitude of the residue curve lied between $+21 \%$ and $-39 \%$ at the flow rate $8 \mathrm{ml} \mathrm{min}^{-1}$. Under lower flow rates, they showed Gaussian-like profiles, judging from the asymmetric factors in Table 2 . The amplitude of the residue curve lied between $+9 \%$ and $-12 \%$ at the flow rate $0.5 \mathrm{ml} \mathrm{min}^{-1}$. The shape difference between the observed temporal peaks and the corresponding Gaussian peaks became greater at the higher flow rates due to higher dispersion $[25,42,43]$. The results echoed with the outcome in Section 4.1 and also demonstrated the influence of the temporal effect on the FIA signal under different flow rates.

Brooks et al. [44] took the second moment (variance) of peaks to analyze the characteristic of the FIA response curves. Under higher flow rate, the second moment raised and resulted in higher peak skewness from the Gaussian shape. Their results also implied that the temporal effect became greater with the flow rate although without direct evidence. 

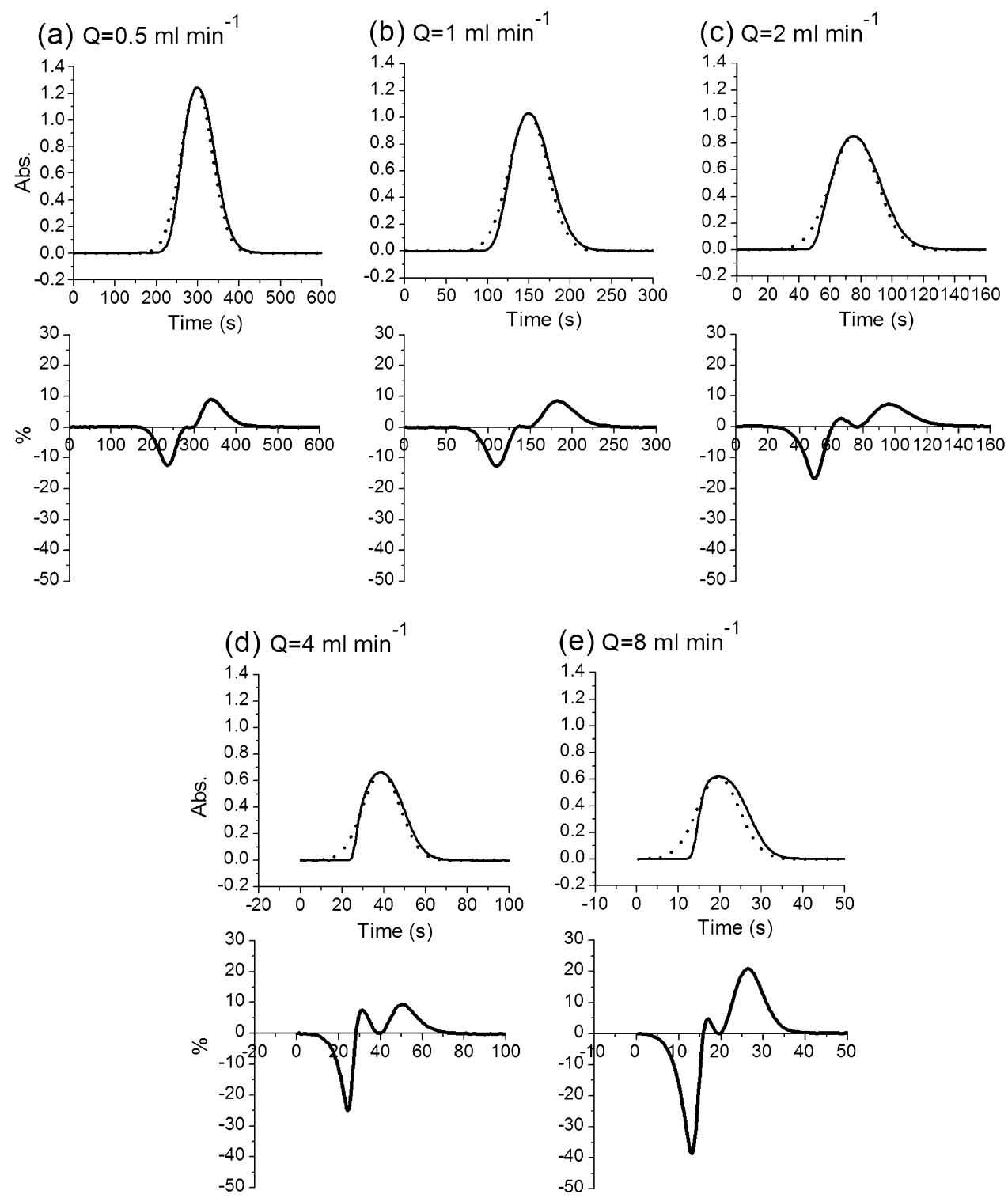

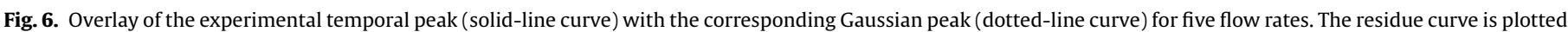
under each peak pair.

\subsection{The temporal effect analyzed by the temporal and spatial data}

The asymmetry factors of the spatial profiles, $A_{f(s)}$, for all five experiments are plotted as a function of longitudinal position, shown in Fig. 7(a). For relatively lower flow rates, namely, 0.5, and $1 \mathrm{ml} \mathrm{min}{ }^{-1}$, the $A_{f(s)}$ for all the five spatial peaks were very close to 0 , while the asymmetry factors for their corresponding temporal peaks, shown in Fig. 7(b), were all significantly greater than 0 . As can be seen, the asymmetry factors as a function of flow rate for the spatial peaks and the temporal peaks were in completely opposite trends. This outcome revealed that the temporal effect should be a major factor in producing tailing peaks. As the flow rates increased (i.e., for 2, 4 and $8 \mathrm{ml} \mathrm{min}^{-1}$ ), the spatial peaks became frontal. The reason why the spatial peaks became frontal for higher flow rates remains unknown in the present; however, their temporal counterparts still showed significant tailing, as shown in Fig. 7(b). In other words, even when a spatially frontal peak was moving in the FIA tubing, a tailing peak would be observed using a fixed singlechannel detector. The asymmetry factors of the temporal peaks with higher flow rates were larger than those with lower flow rates, as shown in Fig. 7(b) and Table 2. It was also noted that the $A_{f(s)}$ varied only in a small range for each individual flow rate no matter where the spatial peaks were monitored along the entire tubing. While the temporal peak propagated towards the tubing outlet, the asymmetry factors were gradually reduced, similar to the results observed using a conventional single-channel detector [24]. The temporal effect gradually decreased while the peak distribution became more spread out. The analyte dispersion apparently was reduced as a function of time under this circumstance. Although perfect Gaussian peaks were never observed in any of the obtained spatial peaks, their asymmetry factors were all very close to zero, that is, their peak shapes remained Gaussian-like only with little skewness.

Assuming that the deviation from the Gaussian profile for the spatial peaks (refrained from the temporal effect) occurred completely due to the Poiseuille effect, this effect apparently affected the peak skewness only very slightly for the spatial peaks. The skewness of the temporal peaks was, however, subjected to both the Poiseuille and the temporal effects. Since the Poiseuille effect only 
(a) Asymmetric factors for the spatial peaks

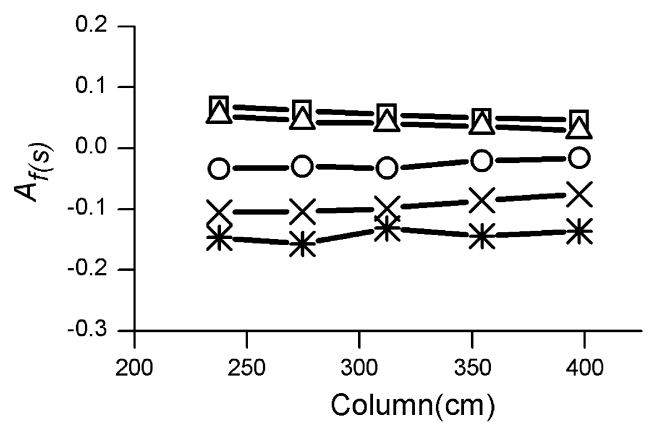

(b) Asymmetric factors for the temporal peaks

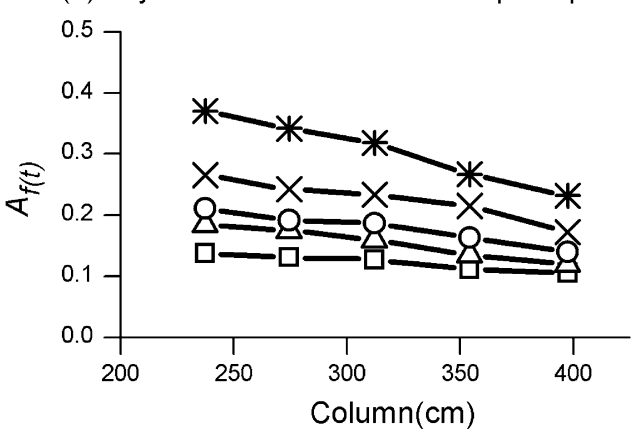

Fig. 7. Asymmetric factors of the (a) spatial peaks and (b) temporal peaks plotted as a function of the longitudinal position in which the peaks were obtained. $\square$ : $0.5 \mathrm{ml} \mathrm{min}^{-1} ; \triangle: 1 \mathrm{ml} \mathrm{min}^{-1} ; \bigcirc: 2 \mathrm{ml} \mathrm{min}^{-1} ; \times: 4 \mathrm{ml} \mathrm{min}^{-1} ;{ }^{*}: 8 \mathrm{ml} \mathrm{min}^{-1}$.

influenced mildly, the asymmetry for the temporal peaks should mainly result from the temporal effect.

\section{Conclusions}

It should be noted that tailing FIA signals obtained conventionally using a single-channel detector are perfectly fine for quantitative analyses. Nevertheless, we verify in this study that the skewness for FIA peaks occurs mainly because of the temporal effect and not the Poiseuille effect. Even a frontal spatial peak would give a greatly skewed tailing peak on the time coordinate due to the temporal effect. Although both spatial and temporal effects have been proposed previously to explain FIA tailing peaks [14-16,20-24,43,44], we provided a direct observation to examine the effects in this study. The temporal effect is also considered to affect all kinds of flowing analytical signals, and the same perception has been extended in the development of a new Parcel model to simulate chromatographic process $[45,46]$. In addition to a series of theoretical investigations by Pai and coworkers, this study helps analyze the temporal effect and provide a simple technical approach for the future studies.

\section{Acknowledgement}

The financial support from the National Science Foundation (NSC 94-2113-M-009-018) of Taiwan is greatly appreciated.

\section{References}

[1] J. Ruzicka, E.H. Hansen, Anal. Chim. Acta 78 (1975) 145.

[2] J.P. Foley, J.G. Dorsey, Anal. Chem. 55 (1983) 730.

[3] J.P. Foley, J.G. Dorsey, J. Chromatogr. Sci. 22 (1984) 40.

[4] D. Hanggi, P. Carr, Anal. Chem. 57 (1985) 2394.

[5] J.P. Foley, Anal. Chem. 59 (1987) 1984.

[6] D. Betteridge, C.Z. Marczewski, A.P. Wade, Anal. Chim. Acta 165 (1984) 227.

[7] C.D. Crown, H.W. Levin, D. Betteridge, A.P. Wade, Anal. Chim. Acta 194 (1987) 49.

[8] P.D. Wentzell, M.R. Bowdridge, E.L. Taylor, C. MacDonald, Anal. Chim. Acta 278 (1993) 293.

[9] J.M. Reijn, W.E. VanDer Linden, H. Poppe, Anal. Chim. Acta 114 (1980) 105.

[10] R. Tijssen, Anal. Chim. Acta 114 (1980) 71.

[11] J.T. Vanderslice, K.K. Stewart, A.G. Rosenfeld, D.J. Higgs, Talanta 28 (1981) 11

[12] J. Ruzicka, E.H. Hansen, Flow Injection Analysis, 2nd ed., Wiley, New York, Chichester, Brisbane, Toronto, Singapore, 1988.

[13] D.A. Skoog, F.J. Holler, T.A. Nieman, Principles of Instrumental Analysis, 5th ed., Saunders College Pub./Harcourt Brace College Publishers, Philadelphia/Orlando, 1998.

[14] S. Olsen, J. Ruzicka, E.H. Hansen, Anal. Chim. Acta 136 (1982) 101.

[15] J. Ruzicka, Phil. Trans. R. Soc. Lond. A 305 (1982) 645.

[16] J. Ruzicka, E.H. Hansen, Anal. Chim. Acta 145 (1983) 1.

[17] D.C. Stone, J.F. Tyson, Anal. Proc. 23 (1986) 23.

[18] D.C. Stone, J.F. Tyson, Analyst 4 (1987) 515.

[19] S.D. Kolev, E. Pungor, Anal. Chem. 60 (1988) 1700

[20] E. Glueckauf, Trans. Faraday Soc. 51 (1955) 34.

[21] R. de Levie, Principles of Quantitative Chemical Analysis, International Editions, McGraw-Hill, New York, 1997.

[22] J.C. Giddings, Unified Separation Science, John Wiley \& Sons, New York, 1991.

[23] A. Felinger, J. Chromatogr. A 1148 (2007) 260

[24] S.C. Pai, J. Chromatogr. A 950 (2002) 271.

[25] G. Taylor, Proc. R. Soc. London, Ser. A 219 (1953) 186.

[26] G. Taylor, Proc. R. Soc. London, Ser. A 223 (1954) 446

[27] R. Aris, Proc. R. Soc. London, Ser. A 235 (1956) 67.

[28] R. Aris, Chem. Eng. Sci 9 (1959) 266.

[29] L. Lapidus, N.L. Amundson, J. Phys. Chem. 56 (1952) 984.

[30] C.E. Evans, V.L. McGuffin, Anal. Chem. 60 (1988) 573.

[31] K.L. Rowlen, K.A. Duell, J.P. Avery, J.W. Birks, Anal. Chem. 61 (1989) 2624

[32] K.L. Rowlen, K.A. Duell, J.P. Avery, J.W. Birks, Anal. Chem. 63 (1991) 575.

[33] D.G. Gelderloos, K.L. Rowlen, J.W. Birks, Anal. Chem. 58 (1986) 900.

[34] J. Wu, J. Pawliszyn, Anal. Chem. 64 (1992) 224.

[35] J. Wu, C. Tragas, A. Watson, J. Pawliszyn, Anal. Chim. Acta 383 (1998) 67.

[36] Z. Liu, J. Pawliszyn, Anal. Chem. 77 (2005) 165.

[37] R.A. Shalliker, B.S. Broyles, G. Guiochon, J. Chromatogr. A 826 (1998) 1.

[38] R.A. Shalliker, B.S. Broyles, G. Guiochon, J. Chromatogr. A 855 (1999) 367

[39] R.A. Shalliker, B.S. Broyles, G. Guiochon, Anal. Chem. 72 (2000) 323.

[40] S.H. Lin, T. Yu, A. Sheu, D.J. Yang, S.C. Pai, J. Chromatogr. A 1201 (2008) 128.

[41] S.V. Romanenko, A.G. Stromberg, T.N. Pushkareva, Anal. Chim. Acta 580 (2006) 99.

[42] A. Shankar, A.M. Lenhoff, AIChE. J 35 (1989 2048).

[43] S.C. Pai, Y.H. Lai, L.Y. Chiao, T. Yu, J. Chromatogr. A 1139 (2007) 109.

[44] S.H. Brooks, D.V. Leff, M.A. Hernandez Torres, J.G. Doresy, Anal. Chem. 60 (1988) 2737.

[45] S.C. Pai, J. Chromatogr. A 988 (2003) 233.

[46] S.C. Pai, C.S. Chern, L.Y. Chiao, J. Chromatogr. A 1018 (2003) 125. 